\title{
Battling with environments: drug delivery to target tissues with particles and functional biomaterials
}

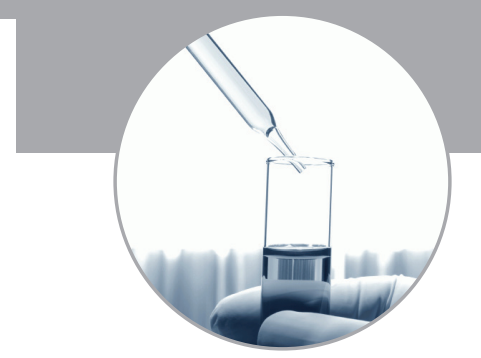

Recent years have seen a growing interest in drug-delivery technology as an enabling tool for complicated pharmacological activities. At the same time, this field has faced as many challenges as successes in translating novel ideas into clinical benefits. The Laboratory for Therapeutic Particles and Biomaterials Engineering at Purdue University (IN, USA) has striven to identify the current challenges in drug delivery and find solutions through the design of new drug-delivery systems. We develop new inhalable formulations for drug and gene delivery for cystic fibrosis patients, simple particle platforms for inhalable drug delivery, anion-resistant nonviral gene vectors, tumor-targeted nanoparticle systems, and hydrogel-based therapeutics. Through expanded collaborations with researchers in medicine and related disciplines, we strive to contribute to advancing the drug delivery field in a clinically relevant manner.

The Laboratory for Therapeutic Particles and Biomaterials Engineering is a young laboratory in the Department of Industrial and Physical Pharmacy at Purdue University (IN, USA). The laboratory consists of a group of students and research associates with backgrounds in pharmacy, chemistry, medicine, chemical engineering, materials engineering and biomedical engineering. Since the laboratory opened in 2007, we have striven to develop new drug-delivery systems and biomaterials that enable safe, efficient and clinically relevant delivery of drugs, genes, and/or cells in a target-specific manner. We are currently developing inhalable dry particles for the treatment of chronic pulmonary diseases; nanoparticle formulations that would enhance tumor-specific drug distribution; and chitosan derivatives as functional excipients. Our research topics are at the interface of biology and chemistry and have attracted several collaborators at Purdue and other institutions.

\section{Inhalable drug formulations for cystic fibrosis therapy}

Inhalable microparticles are an attractive treatment option for chronic pulmonary diseases such as cystic fibrosis (CF), asthma or chronic obstructive pulmonary disease (COPD) because they can provide efficient local medication with minimal systemic side effects, a prolonged therapeutic effect and an easy mode of administration. Recent advances in particle technology have overcome a number of hurdles in formulating microparticles with favorable aerodynamic properties. However, existing technologies do not adequately address the biological barriers specific to pulmonary diseases.
We recognize that the mucus layer on the lung epithelium is a significant barrier for pulmonary drug delivery, especially in CF therapy and obstructive lung diseases [1]. CF is an autosomal recessive disorder caused by mutations in the $\mathrm{CF}$ transmembrane conductance regulator (CFTR) gene, which is responsible for control of epithelial ion transport and water movement. CFTR malfunction results in accumulation of thickened, stationary mucus in the airway, chronic bacterial infection and inflammation and, ultimately, end-stage respiratory failure due to progressive lung destruction. The thick, tenacious CF mucus also presents a significant challenge for effective inhalational delivery of many therapeutic agents [1]. For effective inhalational CF therapy there are at least two avenues of research to explore: enabling penetration and even distribution of drugs within the mucus (antibiotics or anti-inflammatory drugs); and enabling penetration of drugs across the mucus to reach the underlying epithelium (ion-transport regulators or gene therapeutics).

With these challenges in mind, our group developed an inhalable dry-particle system codelivering recombinant human deoxyrybonucleotidase (DNase), which is a mucolytic, and ciprofloxacin, which is an antibiotic [2]. The approach was based on the hypothesis that co-delivery of a mucolytic with an antibiotic decreases the viscoelastisticity of mucus and enhances penetration of the antibiotic. The particles co-delivering ciprofloxacin and DNase decreased the viscoelasticity of the artificial mucus, which resembled the CF mucus in chemical composition and rheological properties. Moreover, this particle system was able to kill the bacteria contained

\section{Yoon Yeo}

Department of Industrial \& Physical Pharmacy, College of Pharmacy, and Weldon School of Biomedical

Engineering, Purdue University, 575 Stadium Mall Drive, West Lafayette, IN 47907, USA

Tel.: + I 7654969608

Fax: + 7654946545

E-mail:yyeo@purdue.edu 


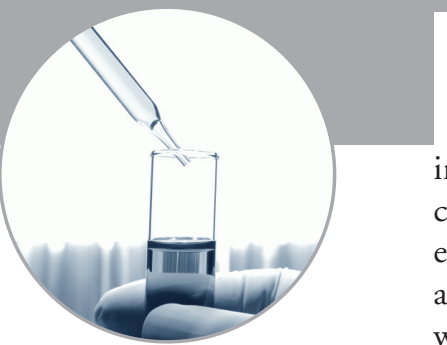

in the mucus more efficiently than the particles containing ciprofloxacin alone (FIgure I). This enhancement of antibacterial efficiency was attributed to the mucolytic activity of DNase, which promoted penetration of the dry particles into the artificial mucus and efficient dissolution and diffusion of ciprofloxacin. This study suggested that co-delivery of antibiotics and DNase using a single inhalable particle system would be a promising strategy for local antibacterial therapy in the CF airway.

Our goal is to expand this principle to delivery of nucleic acid therapeutics to CF lungs to repair the genetic defects. In an attempt to deliver a gene-polymer complex through CF mucus using a similar principle, we are currently exploring alternative excipients that are compatible with nucleic acids, form inhalable particles, and have the ability to enhance gene-complex transport across the mucus. Our research in CF drug delivery is carried out in collaboration with Bumsoo Han from the School of Mechanical Engineering at Purdue and Michael D Tsifansky from the Department of Pediatrics at Lutheran General Children's Hospital (IL, USA).

\section{Inhalable drug-delivery platforms}

A significant challenge in engineering inhalable particles is to ensure that a high, consistent fraction of particles deposit in the lower airways. In their seminal work in the late 1990s, Edwards et al. proposed a porous particle system,

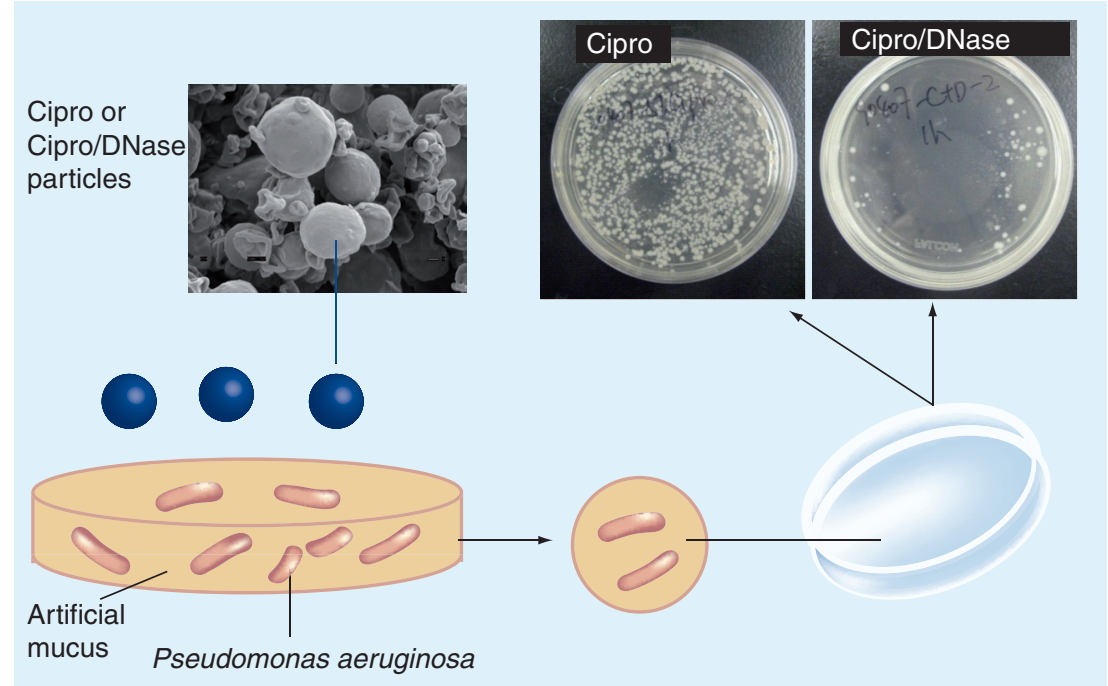

Figure 1. Antibacterial activities of spray-dried particles. Pseudomonas aeruginosa, the major microorganism in the cystic fibrosis airway, was grown in artificial mucus and treated with particles containing ciprofloxacin only (Cipro) or ciprofloxacin and DNase (Cipro/DNase) for $1 \mathrm{~h}$. A total of $10 \mu \mathrm{l}$ of the diluted mucus was plated on a tryptic soy agar plate and incubated overnight. Cipro and Cipro/DNase contained the same amount of ciprofloxacin. DNase itself did not have antibacterial activity. with a large geometric particle size (thereby avoiding aggregation and phagocytic uptake) but with desirable aerodynamic properties $[3,4]$. These large porous particles have typically been produced by inclusion in the double-emulsion method of osmogens or porogens, which generate pores via mass transport between the particle and continuous phases. A disadvantage of these methods is that the pore formation process can interfere with efficient drug encapsulation.

To overcome this problem, we used an effervescent salt as an alternative porogen. Poly(lacticco-glycolic acid) (PLGA) microparticles (average diameter, $10-20 \mu \mathrm{m}$ ) were made by the double emulsion method. To impart favorable aerodynamic properties, ammonium bicarbonate $(\mathrm{ABC})$ was included in the internal aqueous phase as an effervescent porogen [5]. ABC induced a highly porous structure (FIGURE 2 ) in the PLGA particles as it decomposed into ammonia and carbon dioxide. Aerodynamic properties of the resulting particles were easily controlled by manipulating the amount of $\mathrm{ABC}$ and the geometric particle size. Two model drugs, lysozyme and doxorubicin $\mathrm{HCl}$, representing therapeutic macromolecules and small molecularweight drugs, respectively, were encapsulated with high efficiency ( $100 \%$ for both drugs). The high encapsulation efficiency is attributable to the fact that the pore formation relies on the effervescence of gas products rather than on diffusional mass exchange. Gas formation also accelerates solidification of the polymer phase, further increasing the encapsulation efficiency. We are currently evaluating the long-term effect of the porous particles on lung tissues.

Another inhalable particle platform we found particularly useful for the delivery of macromolecules is spray-dried lactose particles with an optimal content of leucine. Basic fibroblast growth factor (bFGF) is a protein therapeutic deemed promising for the therapy of obstructive lung diseases [6], but the development of an inhalable formulation of bFGF is constrained by the relatively poor stability of the protein. It was not compatible with the excipient and solvent system routinely used for other proteins [7]. On the other hand, bFGF prepared in a spraydried matrix of lactose and leucine maintained biological integrity and demonstrated desirable aerodynamic properties [8]. In recognition of the unique contribution of leucine to particle dispersion, we are currently investigating the role of leucine and other hydrophobic amino acids in the formation of spray-dried particles. We anticipate that the insights obtained from 
this study will benefit not only the inhalable particle formulations but also particle engineering for solid dosage forms.

\section{Anion-resistant nonviral gene vector}

Nonviral vectors have been considered a promising alternative to viral vectors for the delivery of gene therapeutics. A critical limitation of nonviral vectors, which are mostly based on cationic lipids or polymers, is that their gene transfection efficiency diminishes greatly in the anionic environment prevalent in the body. Cellular toxicity of cationic polymers is another challenge that hampers in vivo application of nonviral vectors. We recently reported that a ternary gene-polymer complex consisting of hyaluronic acid (HA), disulfide-crosslinked polyethyleneimine (PEI), and plasmid DNA was relatively nontoxic and achieved a significantly higher transfection efficiency than other polymer systems, especially in the presence of serum (FIgure 3) [9]. This welcome result is attributable to several features of the ternary system. First, the disulfide-crosslinked PEI degrades into smaller pieces in the cells, avoiding the toxic effect of high-molecular weight PEI. Second, HA protects the gene-polymer complex in the extracellular environment and at the early stage of intracellular trafficking. Third, HA and disulfide-crosslinked PEI cooperate to facilitate a timely unpacking of DNA from the complex. Due to its unique ability to protect genes in the anionic environment, we expect that the ternary system will be advantageous for the pulmonary delivery of therapeutic genes to CF airway epithelia covered with pathological mucus. We are currently developing inhalable particle forms of the ternary complex for gene delivery to the CF airways.

\section{Tumor-targeted drug delivery}

In developing safe and effective chemotherapy, it is crucial to engineer a targeted drug-delivery system that can selectively deliver antiproliferative drugs to tumor cells without affecting normal cells. While extensive efforts are made to enhance recognition of the drug carriers by tumor tissues (i.e., 'active targeting'), the targeting effect is mostly achieved by the enhanced permeability and retention effect, irrespective of the strategy [10]. This limitation of the active targeting strategy is due, in part, to the diversity and heterogeneity of the tumor cells. Moreover, we recently found that PLGA nanoparticles, a popular platform for tumor-targeted drug delivery, could leach out the entrapped drugs in an uncontrolled manner in the presence of hydrophobic components such as serum proteins or lipids [11]. The uncontrolled drug release in circulation (not in a buffer) is likely another key reason for the limited targeting effect. Therefore, we aim to develop new nanocarrier systems that remain inert without releasing drugs in normal tissues, but are transformed into a cell-interactive form by common tumor microenvironmental features such as $\mathrm{pH}$. With this goal in mind, we developed a new biocompatible chitosan
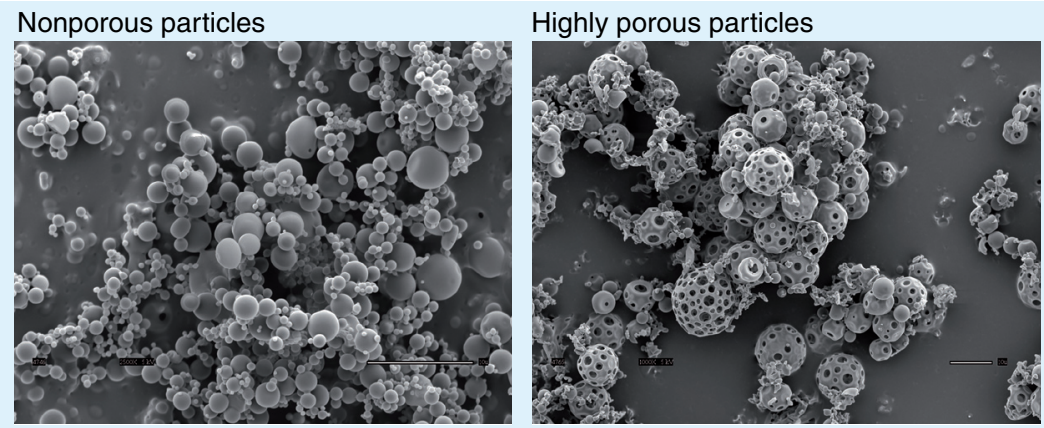

Figure 2. Scanning electron microscopic images of nonporous microparticles and highly porous microparticles prepared with an effervescent porogen. Both particles were made of poly(lactic-co-glycolic acid) (lactide: glycolide $=50: 50$, acidic end group, inherent viscosity $=0.18 \mathrm{dl} / \mathrm{g}$ ). Scale bar $=10 \mu \mathrm{m}$.
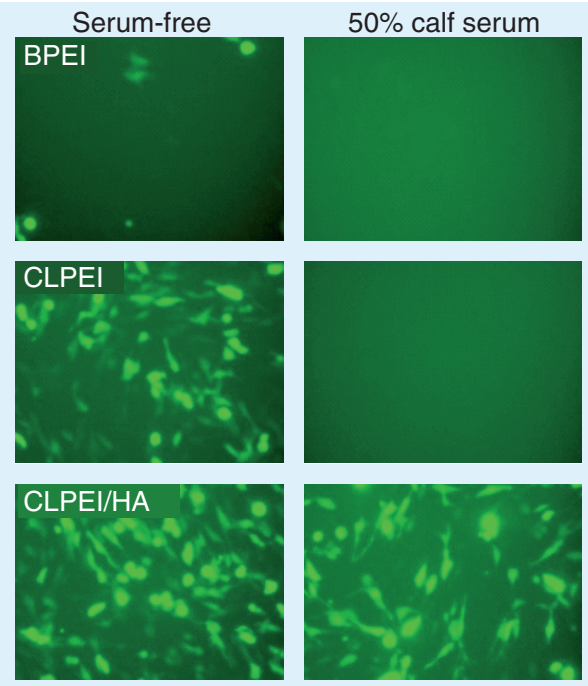

Figure 3. Fluorescence images of NIH/3T3 fibroblasts transfected with plasmid DNA encoding green fluorescent protein complexed with branched

polyethyleneimine, disulfide-CLPEI, or CLPEI and HA, in serum-free medium and $\mathbf{5 0} \%$ calf serum.

Original magnification: $100 x$

CLPEI: Crosslinked polyethyleneimine;

HA: hyaluronic acid.

Reprinted with permission from [9]. 


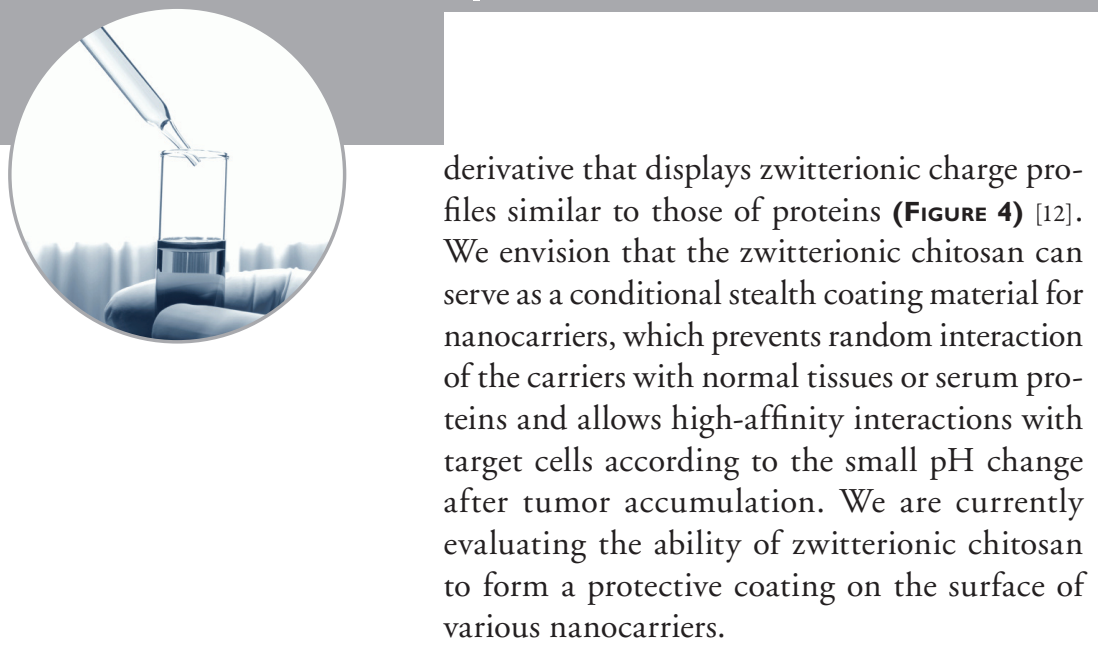

\section{In situ crosslinkable hydrogels}

Hydrogels are attractive materials for a variety of biomedical applications owing to their permeability, flexibility, biocompatibility, and

(A)

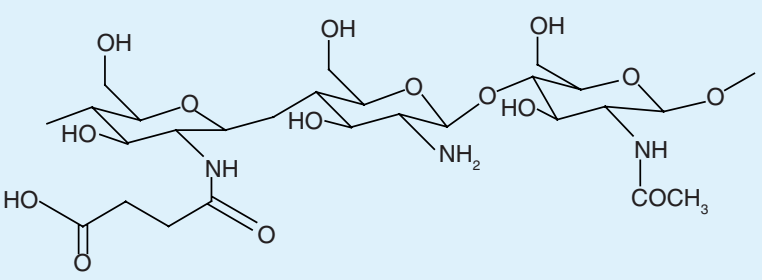

(B)

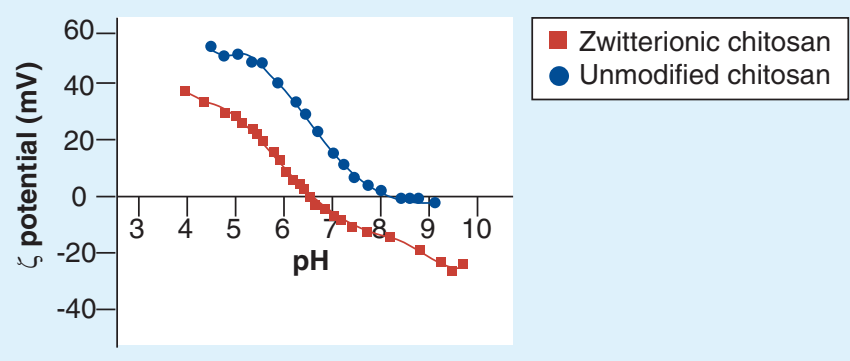

Figure 4. (A) Zwitterionic chitosan prepared by partial amidation of chitosan with succinic anhydride. (B) $\mathrm{pH}$ dependence of $\zeta$ potential of zwitterionic chitosan and unmodified chitosan.
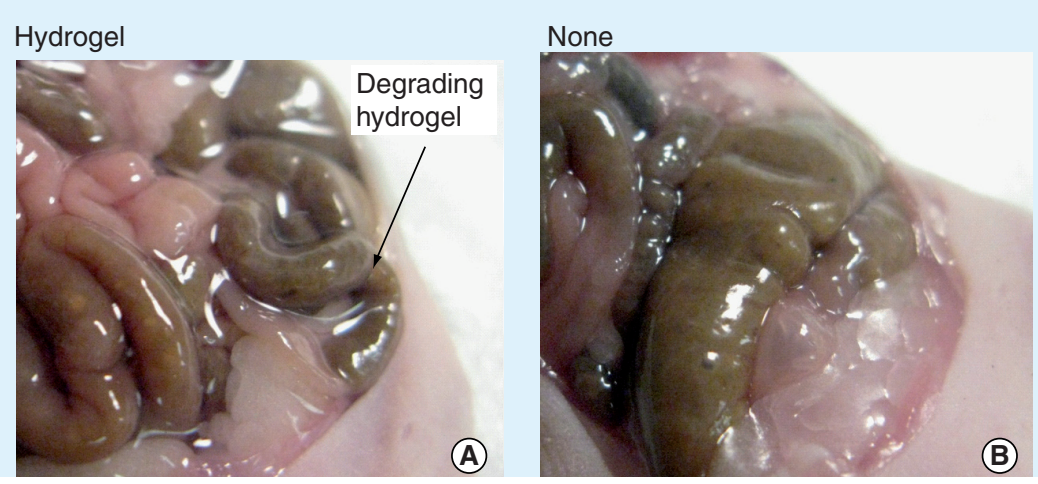

Figure 5. In situ crosslinkable hydrogel as a local drug delivery medium in the peritoneal cavity. (A) Viscous liquid lining the intraperitoneal organs indicates degrading hydrogel, 2 weeks after injection into a nude mouse;

(B) Untreated peritoneal cavity. structural similarity to the extracellular matrix. Our group has studied in situ crosslinkable hydrogels based on HA and chitosan for intraperitoneal drug delivery [13], use as a tissue adhesive [14] or for tissue engineering $[15,16]$. Previously, we reported that a hydrogel was an excellent delivery medium for prolonging the drug residence time and enhancing the pharmacological effect in the peritoneal cavity $[17,18]$. Tissue type plasminogen activator [18] or budesonide [17] delivered via a HA hydrogel to the injured peritoneum showed a dramatic anti-adhesion effect, far exceeding that of the free drug or the hydrogel alone. Capitalizing on the same principle, we are testing whether the hydrogel can serve as a delivery medium of antiproliferative drugs to enhance the anticancer activity against the intraperitoneal malignancies (Figure 5). This study is being conducted in collaboration with Sulma Mohammed from the School of Veterinary Medicine at Purdue.

We are also utilizing the in situ crosslinkable hydrogel for assisting in the fusion of severed peripheral nerves or spinal cord. While no effective therapies to treat nerves that are completely severed currently exist, it is shown that the function of transected nerves can be immediately restored ex vivo with appropriate efforts to reconnect the nerves [19]. We are utilizing photocrosslinkable chitosan hydrogels with improved mechanical strengths as bioadhesives that substitute fibrin glue. This research is a collaborative effort with Riyi Shi from the Department of Basic Medical Sciences at Purdue.

\section{Future perspective}

Drug-delivery systems are now considered not only a way of reducing the frequency of medications but also an enabling tool for complicated pharmacological activities. At the same time, this field has faced as many challenges as expectations in translating novel ideas into clinical benefits. Our group aims to identify and address critical challenges that have hampered the clinical translation of advanced drug-delivery systems and functional biomaterials. The mucus barrier in the CF airways, limited access of drug carriers to target tissues, physical and chemical instability of carriers and the lack of means to eradicate refractory and/or metastatic tumors are the main challenges our group has tried to take on through rational design of new drug-delivery systems. Future studies in CF drug delivery will examine the efficacy of inhalable drug/gene delivery systems in relevant disease models. We will continue to develop the conditionally activated nanocarriers for tumor-specific drug 
delivery, responsive to a wide variety of cues including $\mathrm{pH}$, proteinases and other biomarkers specific to the tumor microenvironment. Using biocompatible in situ crosslinkable hydrogels and new nanocarriers, we will also explore the potential of local chemotherapy in the context of intraperitoneal malignancies such as ovarian cancer and peritoneal carcinomatosis. Toward this goal, we anticipate expanding our collaborations with researchers in medicine, biology, chemistry and engineering.

Financial \& competing interests disclosure The Laboratory for Therapeutic Particles and Biomaterials Engineering at Purdue University (IN, USA) has been supported by NIH R21 CA135130, College of Pharmacy at
Purdue; Seed grant from the Lilly Endowment, Inc., to the College of Pharmacy at Purdue; Purdue Research Foundation, Showalter Trust Award, Indiana State Department of Health; Indiana CTSI Pre-doctoral Training Fellowship; Cystic Fibrosis Foundation; $3 M$ NonTenured Faculty Grant; AACP New Investigator Award, AAPS New Investigator Grant Award in Pharmaceutics and Pharmaceutical Technologies, Dong-A Pharmaceutical Co., Ltd (Korea), and DKC Corporation (Korea). The author has no other relevant affiliations or financial involvement with any organization or entity with a financial interest in or financial conflict with the subject matter or materials discussed in the manuscript apart from those disclosed.

No writing assistance was utilized in the production of this manuscript.

\section{Bibliography}

1 Hanes J, Demeester J. Drug and gene delivery to mucosal tissues: the mucus barrier. Adv. Drug Deliv. Rev. 61(2), 73-74 (2009).

2 Yang Y, Tsifansky M, Wu C-J, Yang HI, Schmidt G, Yeo Y. Inhalable antibiotic delivery using a dry powder co-delivering recombinant deoxyribonuclease and ciprofloxacin for treatment of cystic fibrosis. Pharm. Res. 27(1), 151-160 (2010).

3 Edwards DA, Hanes J, Caponetti G et al. Large porous particles for pulmonary drug delivery. Science 276(5320), 1868-1871 (1997).

4 Edwards DA, Ben-Jebria A, Langer R. Recent advances in pulmonary drug delivery using large, porous inhaled particles. J. Appl. Physiol. 85(2), 379-385 (1998).

5 Kim TK, Yoon JJ, Lee DS, Park TG. Gas foamed open porous biodegradable polymeric microspheres. Biomaterials 27(2), 152 (2006).

6 Jeon S, Lee C, Oh M et al. Recombinant basic fibroblast growth factor inhibits the airway hyperresponsiveness, mucus production, and lung inflammation induced by an allergen challenge. J. Allergy Clin. Immunol. 119(4), 831-837 (2007).
7 Park SW, Shin S, Kim CH et al. Differential effects of insufflated, subcutaneous, and intravenous growth hormone on bone growth, cognitive function, and NMDA receptor subunit expression. Endocrinology 151(9), 4418-4427 (2010).

8 Ibrahim BM, Jun SW, Lee MY, Kang SH, Yeo Y. Development of inhalable dry powder formulation of basic fibroblast growth factor. Int. J. Pharm. 385, 66-72 (2010).

9 Xu P, Quick G, Yeo Y. Gene delivery through the use of a hyaluronate-associated intracellularly degradable cross-linked polyethyleneimine. Biomaterials 30 (29), 5834-5843 (2009).

10 Gullotti E, Yeo Y. Extracellularly activated nanocarriers: a new paradigm of tumor targeted drug delivery. Mol. Pharm. 6(4), 1041-1051 (2009).

11 Xu P, Gullotti E, Tong L et al. Intracellular drug delivery by poly(lactic-co-glycolic acid) nanoparticles, revisited. Mol. Pharm. 6(1), 190-201 (2009).

12 Xu P, Bajaj G, Shugg T, Van Alstine WG, Yeo Y. Zwitterionic chitosan derivatives for $\mathrm{pH}$-sensitive stealth coating. Biomacromolecules 11(9), 2352-2358 (2010).

13 Bajaj G, Yeo Y. Drug delivery systems for intraperitoneal therapy. Pharm. Res. 27(5), 735-738 (2010)
14 Rickett T, Amoozgar Z, Sun W, Yeo Y, Shi R. A photo-crosslinkable chitosan hydrogel for peripheral nerve anastomosis. Proceedings of the 2009 2nd International Conference on Biomedical Engineering and Informatics. J. Med. Syst. 1-4, 1078-1082 (2009).

15 Rask F, Dallabrida SM, Ismail NS et al. Photocrosslinkable chitosan modified with angiopoietin-1 peptide, QHREDGS, promotes survival of neonatal rat heart cells. J. Biomed. Mater. Res. A 95(1), 105-117 (2010).

16 Yeo Y, Geng W, Ito T, Kohane DS, Burdick J, Radisic M. A photocrosslinkable hydrogel for myocyte cell culture and injection. J. Biomed. Mater. Res. Part B Appl. Biomater. 81(2), 312-322 (2006).

17 Yeo Y, Adil M, Bellas E, Astashkina A, Chaudhary N, Kohane DS. Prevention of peritoneal adhesions with an in situ crosslinkable hyaluronan hydrogel delivering budesonide. J. Control. Release 120(3), 178-185 (2007).

18 Yeo Y, Bellas E, Highley CB, Langer R, Kohane DS. Peritoneal adhesion prevention with an in situ cross-linkable hyaluronan gel containing tissue-type plasminogen activator in a rabbit repeated injury model. Biomaterials 28(25), 3704-3713 (2007).

19 Shi R, Borgens RB, Blight AR. Functional reconnection of severed mammalian spinal cord axons with polyethylene glycol. J. Neurotrauma 16(8), 727-738 (1999). 